\title{
Programmable pulsewidth control loop (PWCL) in dual-slope combination
}

\author{
H. S. Mo, B. K. Moon, and Daejeong Kim ${ }^{\mathrm{a})}$ \\ Department of Electronics Engineering, Graduate School, Kookmin University \\ 861-1 Jeongneung-dong, Seongbuk-gu, Seoul 136-702, Korea \\ a)kimdj@kookmin.ac.kr
}

\begin{abstract}
A new technique to adjust the duty cycle of the output clock by adopting a pulsewidth control loop (PWCL) in dual-slope combination is presented. Correcting both rising and falling slopes simultaneously in their combination leads to a simple way for a wide correction range. Furthermore, the duty cycle can be easily adjusted to a desired value by setting up the programmable current sources in the charge pump. A generic circuit is suggested, and its validity is verified in a $0.13-\mu \mathrm{m}$ CMOS technology under $1.2 \mathrm{~V}$ supply. The simulated results with Spectre exhibit the integral nonlinearity (INL) of less than $2 \%$ for the output duty-cycle range from $6.25 \%$ to $93.75 \%$ with the consideration of process, voltage, and temperature (PVT) variation.
\end{abstract}

Keywords: duty cycle, pulsewidth control loop (PWCL), programmable

Classification: Integrated circuits

\section{References}

[1] F. Mu and C. Svensson, "Pulsewidth control loop in high-speed CMOS clock buffers," IEEE J. Solid-State Circuits, vol. 35, no. 2, pp. 134-141, Feb. 2000.

[2] P. H. Yang and J. S. Wang, "Low-voltage pulsewidth control loops for SOC applications," IEEE J. Solid-State Circuits, vol. 37, no. 10, pp. 1348-1351, Oct. 2002.

[3] W. M. Lin and H. Y. Huang, "A low-jitter mutual-correlated pulsewidth control loop circuit," IEEE Int. Conf. Systems-on-Chip, pp. 301-304, 2003.

[4] S. R. Han, et al., "A Single-Path Pulsewidth Control Loop With a BuiltIn Delay-Locked Loop," IEEE J. Solid-State Circuits, vol. 40, no. 5, pp. 1130-1135, May 2005.

\section{Introduction}

The systems adopting half-rate techniques require a clock with $50 \%$ duty cycle. Unfortunately, the process, voltage, and temperature (PVT) variations may cause its deviation from the desired value. Several pulsewidth control 
loops (PWCL) to tolerate the PVT variations $[1,2,3]$ have been presented. However, the unbalanced variation between two charge pumps may create large jitter $[1,3]$, or an additional overhead of the hardware and power consumption is encountered [2]. Above all things, these works hardly provide any prescribed strategy of adjusting the duty rate. A programmable PWCL utilizing a voltage-controlled delay line (VCDL) in a 3 -state sequential circuit to determine the falling edge of the output clock [4] was reported. It overcomes many drawbacks of the previous works, but the delayed version of the reference clock propagating through the VCDL and flip-flops for correcting the duration still restricts the adjusting range of the duty cycle.

In this paper, a new topology of pulsewidth control loop (PWCL) in a dual-slope combination is proposed without using any variable delay lines as in the prior works $[1,2,3,4]$. Controlling both rising and falling slopes with an efficient combiner provides some fundamental strategies to make any duration rate possible. A typical implementation of the topology is suggested and its performance results are described.

\section{Concept of correcting duty cycle}

Fig. 1 (a) represents a block diagram of the proposed PWCL to take in the wide duty-cycle range of the input $\left(\mathrm{CK}_{\mathrm{I}}\right)$. It consists of a slope modifier, a pulse combiner, and a charge pump. The duty cycle of $\mathrm{CK}_{\mathrm{O}}$ and $/ \mathrm{CK}_{\mathrm{O}}$ is converted to the tuning-line analog voltage, $v_{c}$, which is then fed back to the slope modifier. The duty-cycle information on the current complementary outputs is represented by $v_{c}$ which is updated every iteration, and finally settles down. The variation of circuit parameters such as gate propagation delay, logic threshold voltage, and device drivability would be alleviated through the loop operations, resulting in little long-term effects.

Recognizing that the input and output quantities, $\Phi_{\mathrm{I}}$ and $\Phi_{\mathrm{O}}$, are the excess phases from the ideal position, a linear model to describe the duty-cycle correcting dynamics is shown in Fig. $1(\mathrm{~b})$. In this case, $K_{V C \Phi}$ represents the gain of the correction associated with the slope modifier and the pulse combiner. It would be considered to be a function of the trans-conductance of the current source and the output capacitance in the slope modifier shown in Fig. $1(\mathrm{c}) . I_{B}$ and $C$ are the bias current and the storage capacitance of the charge pump, respectively. The closed-loop gain for the excess phase can be expressed such as

$$
\frac{\Phi_{O}}{\Phi_{I}}(s)=\frac{2 \pi C s}{2 \pi C s+K_{V C \Phi} \cdot I_{B}}
$$

where a dc zero and a left-half plane pole are involved. Therefore, the dynamic correction range and the gain of the in-band correction can be expressed in (2) and (3), respectively.

$$
\begin{aligned}
& \omega_{0}=\frac{K_{V C \Phi} \cdot I_{B}}{2 \pi C} \\
& G \cong \frac{2 \pi C \omega}{K_{V C \Phi} \cdot I_{B}}
\end{aligned}
$$




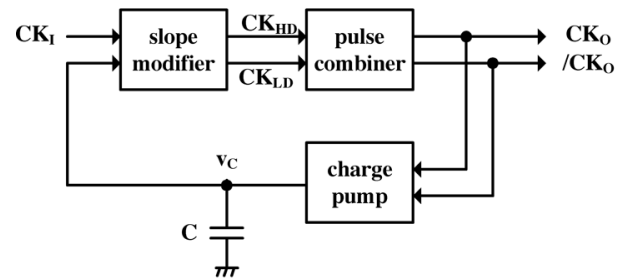

(a)
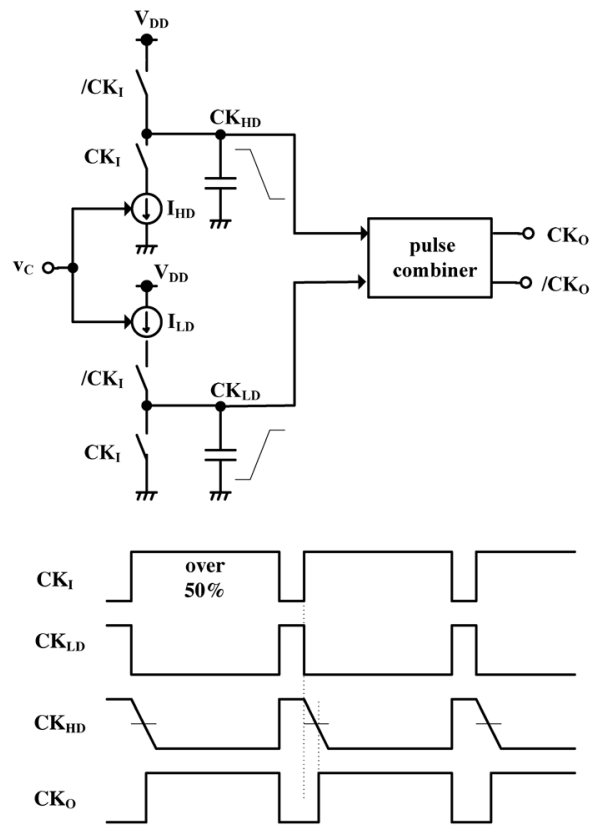

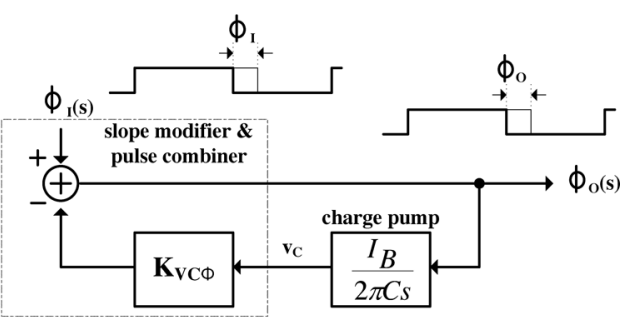

(b)

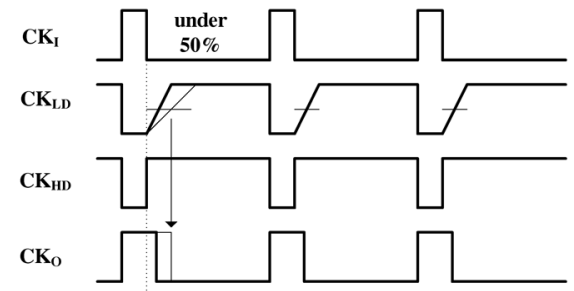

(c)

Fig. 1. Proposed PWCL: (a) block diagram (b) linear model (c) concept of slope modification and composition.

A conceptual diagram for the slope modification followed by a pulse combiner is illustrated with its conceptual operations in Fig. 1 (c). Falling-slope $\left(\mathrm{CK}_{\mathrm{HD}}\right)$ and rising-slope $\left(\mathrm{CK}_{\mathrm{LD}}\right)$ modifiers are placed in parallel, each consisting of complementary switches driven by the input clock, a current source ( $I_{L D}$ or $\left.I_{H D}\right)$ controlled by the analog tuning-line voltage, $v_{c}$, and a capacitor. The pulse combiner driven by both $\mathrm{CK}_{\mathrm{HD}}$ and $\mathrm{CK}_{\mathrm{LD}}$ composes a composite pulse, and then produces the corresponding complementary clocks, $\mathrm{CK}_{\mathrm{O}}$ and $/ \mathrm{CK}_{\mathrm{O}}$.

The basic operation, when the switch resistance is neglected, is as follows. The tuning-line voltage, $v_{c}$, affects the output slopes of the modifier in the opposite direction since it is applied to the opposite types of current sources. If $v_{c}$ decreases, so $I_{H D}$ decreases and $I_{L D}$ increases, the falling slope of $\mathrm{CK}_{\mathrm{HD}}$ becomes slower while the rising slope of $\mathrm{CK}_{\mathrm{LD}}$ becomes faster, and vice versa. The correction mechanisms in the three appreciable cases are suggested when the duty cycle of the input $\mathrm{CK}_{\mathrm{I}}$ is under $50 \%$, over $50 \%$, or near $50 \%$. Under $50 \%$, the time required for $\mathrm{CK}_{\mathrm{LD}}$ from Gnd to reach the logic threshold 
voltage $\left(V_{\text {logic }}\right)$ of the following pulse combiner determines the falling instance of $\mathrm{CK}_{\mathrm{O}}$, expanding the pulse duration. For over $50 \%$, the time for $\mathrm{CK}_{\mathrm{HD}}$ from $V_{D D}$ to $V_{\text {logic }}$ determines the rising instance of $\mathrm{CK}_{\mathrm{O}}$, reducing the pulse duration. Near $50 \%$, the duration is nearly maintained since both edges are moving in the same direction. In all cases, the composition of the edges forms a modified duration depending on the tuning-line voltage, $v_{c}$. The duty-cycle correction should be completed by forming a PWCL, as shown in the block diagram of Fig. 1 (a).

\section{Circuit design and simulation results}

Fig. 2 (a) depicts a CMOS realization of the open-loop path from $\mathrm{CK}_{\mathrm{I}}$ to $\mathrm{CK}_{\mathrm{O}}$ and $/ \mathrm{CK}_{\mathrm{O}}$. The slope modifier consists of current sources, $\mathrm{M}_{1}$ and $\mathrm{M}_{6}$, and complementary switches $\mathrm{M}_{2}, \mathrm{M}_{3}$, and $\mathrm{M}_{4}, \mathrm{M}_{5}$, for $\mathrm{CK}_{\mathrm{HD}}$ and $\mathrm{CK}_{\mathrm{LD}}$, respectively. The pulse combiner consists of four stacked transistors, $M_{7} \sim M_{10}$ followed by a latch. The complementary $\mathrm{CK}_{\mathrm{O}}$ and $/ \mathrm{CK}_{\mathrm{O}}$ are generated via the balanced inverter chains. The rising edge of $\mathrm{CK}_{\mathrm{HD}}$ which always advances that of $\mathrm{CK}_{\mathrm{LD}}$ enables the pull-down path $\left(\mathrm{M}_{8}\right)$ while disabling the pull-up path $\left(\mathrm{M}_{10}\right)$. The rising slope of $\mathrm{CK}_{\mathrm{LD}}$, then, turns on $\mathrm{M}_{7}$, beginning to draw current through $\mathrm{M}_{12}$. Eventually, the logic threshold encountered by the rising slope of $\mathrm{CK}_{\mathrm{LD}}$ is determined by $\mathrm{M}_{7}, \mathrm{M}_{8}$ and $\mathrm{M}_{12}$.

Similarly, the falling edge of $\mathrm{CK}_{\mathrm{LD}}$ and the falling slope of $\mathrm{CK}_{\mathrm{HD}}$ enable the pull-up path through $\mathrm{M}_{9}$ and $\mathrm{M}_{10}$ in this order. Again, the logic threshold encountered by the falling slope of $\mathrm{CK}_{\mathrm{HD}}$ is determined by $\mathrm{M}_{9}, \mathrm{M}_{10}$ and $\mathrm{M}_{11}$. The latch acts to hold the previous value during the instantaneous high-impedance interval from the leading edge to the late slope.

The duty cycle of the output can be adjusted by configuring the binaryweighted current sources in the charge pump, as illustrated in Fig. 2 (b). The tuning-line voltage, $v_{c}$, settles down when the incoming pull-up charge is equal to the outgoing pull-down charge. That is, in the steady state

$$
I_{B 1} \cdot T_{/ C K O}=I_{B 2} \cdot T_{C K O},
$$

where $T_{/ C K O}$ and $T_{C K O}$ are the "high" durations of $/ \mathrm{CK}_{\mathrm{O}}$ and CKo, respectively. If the expression of (4) is substituted into the following definition, the duty cycle could be ultimately controlled with respect to the ratio of $I_{B 1}$ to $I_{B T}$.

$$
\text { duty cycle }=\frac{T_{C K O}}{T_{C K O}+T_{/ C K O}}=\frac{1}{1+\frac{T_{/ C K O}}{T_{C K O}}}=\frac{1}{1+\frac{I_{B 2}}{I_{B 1}}}=\frac{I_{B 1}}{I_{B T}}
$$

Typically, the current segments of $I_{B 1}$ will be allowed to be the same as those of $I_{B 2}$, except for the extra termination current source $I_{u}$. The digital codes from $D_{1}$ to $D_{8}$ control the segments of both $I_{B 1}$ and $I_{B 2}$, pertaining to maintain the total current, $I_{B T}$, constant.

In order to prove the validity of the proposed PWCL, a full transistor-level circuit in a $0.13-\mu \mathrm{m}$ CMOS process under $1.2 \mathrm{~V}$ supply voltage was designed. The Spectre simulation results in Fig. 2 (c) exhibit INL error versus digital 


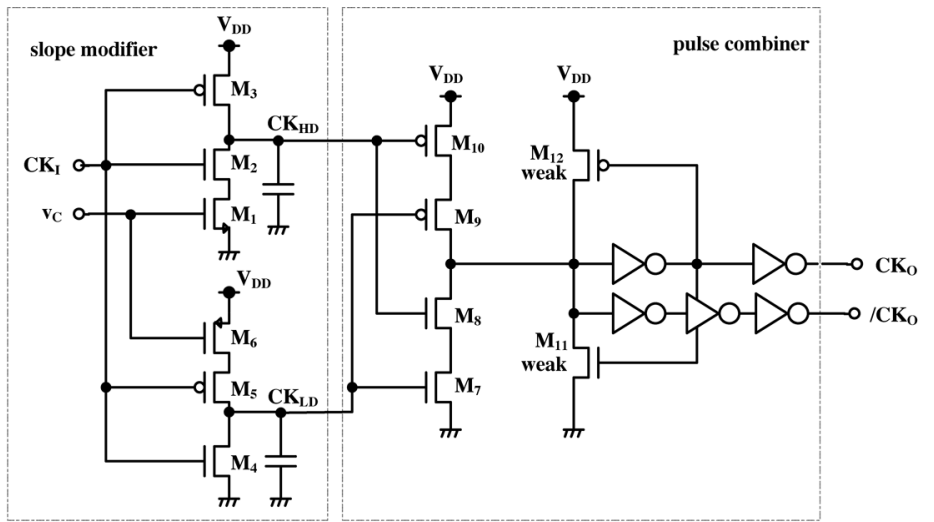

(a)

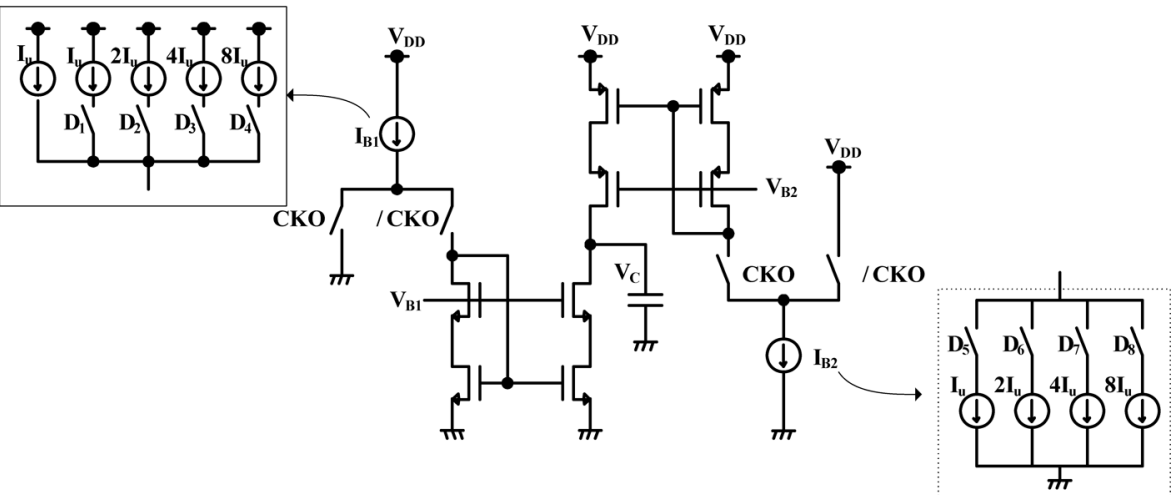

(b)
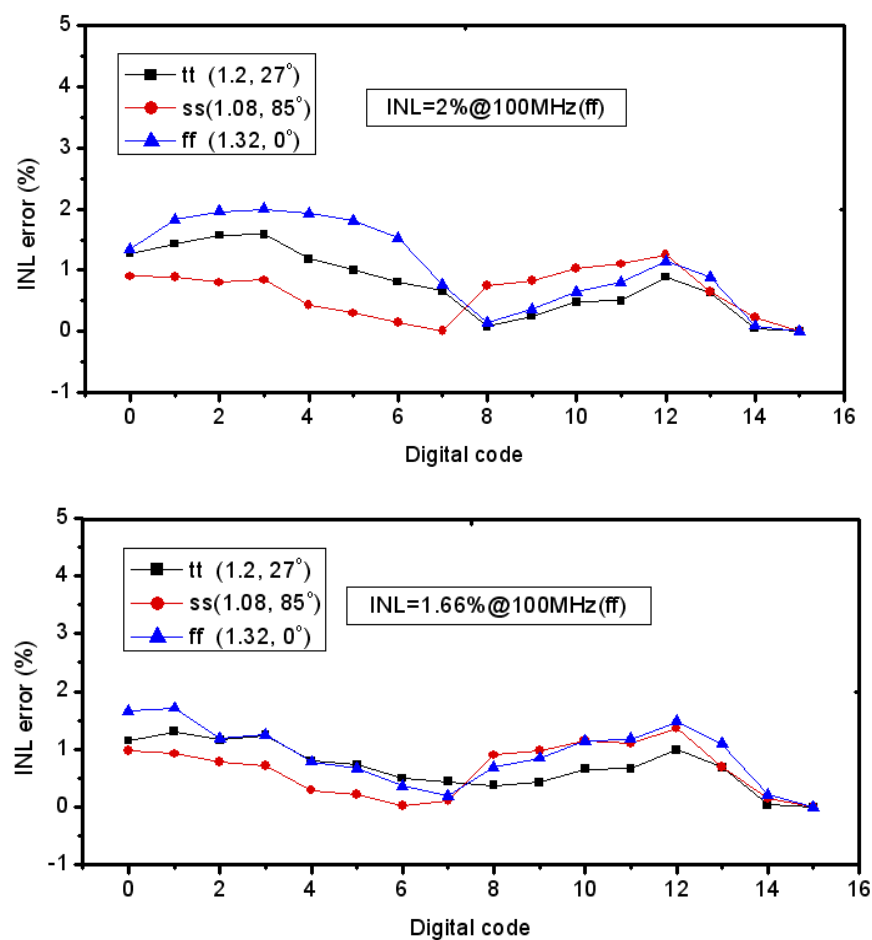

(c)

Fig. 2. Circuit diagrams of (a) slope modifier and pulse combiner, (b) programmable charge pump, and (c) Spectre simulated results with input duty cycle of $20 \%$ and $80 \%$, respectively. 
code with PVT variations when the applied input is at $100 \mathrm{MHz}$ with the duty cycle of $20 \%$ and $80 \%$, respectively. The INL error is observed to increase as the code gets out of the center point of $50 \%$ duty cycle (digital code $=$ 0111). It is primarily due to the finite output impedance of the source device, leading to the mismatch between the pull-up and pull-down currents as the code varies. Table I summarizes the comparison results between the proposed and the conventional PWCLs.

Table I. Performance comparison with prior works.

\begin{tabular}{|c|c|c|}
\hline & This work & {$[4]$} \\
\hline Technology & $0.13-\mu \mathrm{m}$ CMOS & $0.35-\mu \mathrm{m}$ CMOS \\
\hline Supply Voltage & $1.2 \mathrm{~V}$ & $3.3 \mathrm{~V}$ \\
\hline Control Stage Type & Slope modifier & Tri-state control \\
\hline Operating Frequency Range & $\sim 1 \mathrm{GHz}$ & $1 \mathrm{GHz} \sim 1.27 \mathrm{GHz}$ \\
\hline Input Duty Cycle Range & $20 \% \sim 80 \%$ & - \\
\hline Output Duty Cycle Range & $6.25 \% \sim 93.75 \%$ & $35 \% \sim 70 \%$ \\
\hline Active Die Area & $0.25 \times 0.17 \mathrm{~mm}^{2}$ & $0.47 \times 0.3 \mathrm{~mm}^{2}$ \\
\hline Power Consumption & $16 \mathrm{~mW}$ & $150 \mathrm{~mW}$ \\
\hline
\end{tabular}

\section{Conclusion}

A duty-cycle control circuit with small area is developed by adopting a pulsewidth control loop (PWCL) without variable-delay lines. The simultaneous modification of both rising and falling edges of the input in their combination leads to the precise correction over the wide covering range of the input duty cycle. In addition, to achieve a wide programmable range of the output duty cycle, a charge pump with programmable current sources is used. The analysis with a linear model was suggested to investigate the loop dynamics. The proposed PWCL system was designed in a $0.13-\mu \mathrm{m}$ CMOS technology. System parameters and performance results from Spectre simulations are described.

\section{Acknowledgments}

This research was supported by a grant for Research Promotion Program of Kookmin University. 